\title{
Aus dem Vorwort zur ersten Auflage
}

Die erste Anregung zur Abfassung des vorliegenden Buches empfing ich durch mehrfach an mich ergangene Aufforderungen, meine in das Gebiet der Thermodynamik fallenden Abhandlungen gesammelt herauszugeben bzw. zu einer zusammenfassenden Darstellung zu verarbeiten. Wenn auch das erstere Verfahren als das einfachere näher gelegen hätte, so entschied ich mich doch für eine neue Überarbeitung des ganzen Stoffes, weil sich auf diese Weise Gelegenheit bot, mittels einer entsprechenden Erweiterung des behandelten Themas das ganze Gebiet der Thermodynamik in eine einheitliche Darstellung zusammenzufassen. Hierdurch ist dem Werke allerdings der Charakter einer Forschungsarbeit genommen und ihm mehr derjenige eines Lehrbuches gegeben, bestimmt zur Einführung in das Studium der Thermodynamik für jeden, der einen Anfängerkurs in Physik und Chemie durchgemacht hat und mit den Elementen der Differential- und Integralrechnong vertraut ist.

In der bisherigen Entwicklung der Thermodynamik lassen sich deutlich drei voneinander verschiedene Methoden der Forschung unterscheiden. Die erste greift am tiefsten hinein in das Wesen der betrachteten Vorgänge, sie wäre daher, wenn sie sich exakt durchführen lieBe, jedenfalls als die vollkommenste zu bezeichnen. Nach ihr wird die Wärme bedingt durch bestimmte Bewegungen der als diskrete Massen gedachten chemischen Moleküle und Atome, die für gasförmige Körper verhältnismäBig einfache Eigenschaften haben, während sie sich für feste und flüssige Körper bisher nur in rohen Zügen angeben lassen. Diese kinetische Theorie hat seit ihrer Begründung durch Joute, Waterston, Krönig und Clauusius besonders durch MaxweLI und Boltzmann wesentliche Erweiterung und Vertiefung erfahren, scheint aber in ihrer weiteren Entwicklung auf vorläufig 
unüberwindliche Hindernisse zu stoBen, die nicht nur in der hochgradig komplizierten mathematischen Durchführung der angenommenen Hypothesen, sondern vor allen Dingen in prinzipiellen, hier nicht näher zu erörternden Schwierigkeiten bei der mechanischen Deutung der thermodynamischen Hauptsätze begründet sind.

Derartige spezielle Schwierigkeiten vermeidet eine $z$ weite, namentlich von Heцmноцтz ausgebildete, Methode der Thermodynamik, indem sie sich auf die wichtigste Voraussetzung der mechanischen Wärmetheorie beschränkt, daB Wärme auf Bewegung beruht, dagegen auf ein Spezialisieren der Vorstellungen von der Natur dieser Bewegungen zunächst grundsätzlich verzichtet. Dieser Standpunkt ist sicherer als der vorige, er gewährt auch die volle philosophische Befriedigung, die die mechanische Naturauffassung überhaupt liefert, aber der Halt, den er bietet, ist bis jetzt nicht breit genug, um darauf eine Theorie im einzelnen aufzubauen. Alles, was man von ihm ausgehend erreichen kann, ist die Bestätigung einiger allgemeiner schon anderweitig direkt aus der Erfahrung abgeleiteter Gesetze.

Am fruchtbarsten hat sich bisher eine dritte Behandlung der Thermodynamik erwiesen. Diese Methode unterscheidet sich von den beiden zuerst besprochenen wesentlich dadurch, daB sie die mechanische Natur der Wärme nicht in den Vordergrund stellt, sondern, indem sie sich bestimmter Annahmen über das Wesen der Wärme ganz enthält, statt dessen direkt von einigen sehr allgemeinen Erfahrungstatsachen, hauptsächlich von den sogenannten beiden Hauptsätzen der Wärmelehre, ausgeht. Daraus ergeben sich dann auf rein logischem Wege eine große Reihe neuer Sätze der Physik und Chemie, die sich weitgehender Anwendungèn fähig gezeigt und bis jetzt überall ausnahmslos bewährt haben.

Diese letzte Behandlungsart, welche im vorliegenden Werke ausschlieBlich benutzt ist, entspricht wohl am besten dem heutigen Stande der Wissenschaft, sie ist aber kaum als die abschlieBende zu betrachten, sondern wird wahrscheinlich künftig einmal einer mechanischen oder vielleicht auch einer elektromagnetischen Betrachtungsweise Platz machen müssen. Denn wenn es auch eine Zeitlang Vorteil gewähren mag, die einzelnen Wirkungen der Natur: Wärme, Bewegung, Elek- 
trizität usw. zunächst als qualitativ verschieden voneinander einzuführen und die Frage nach ihrer etwaigen Wesensgemeinschaft zu unterdrücken, so wird doch unser durch die Entdeckung des Prinzips der Erhaltung der Energie so mächtig gefördertes Streben nach einer einheitlichen Naturanschaung, sei es auf mechanischer oder auf anderer Grundlage, sich niemals auf die Dauer zurückhalten lassen; würde doch schon heute ein Zurücktreten von der Annahme der Wesensgleichheit aller physikalischen Vorgänge gleichbedeutend sein mit dem Verzicht auf das Verständnis einer Reihe bereits erkannter GesetzmäBigkeiten zwischen verschiedenen Gebieten der Natur. Dann werden selbstrerständlich die hier aus den beiden Hauptsätzen der Wärmelehre abgeleiteten Ergebnisse nicht erschüttert werden, sondern es werden nur diese beiden Sätze nicht mehr selbstständig eingeführt, sondern ihrerseits aus anderen noch allgemeineren Sätzen abgeleitet werden. Es ist aber bis jetzt die Zeit noch nicht abzusehen, in welcher der weite Weg zu diesem Ziel zurückgelegt werden kann.

Berlin, im April 1897 